\title{
IMMUNOHISTOCHEMICAL EXPRESSION OF SONIC HEDGEHOG SIGNALING AND ITS INFLUENCE ON ANGIOGENESIS IN OSTEOSARCOMA AND CHONDROSARCOMA
}

\author{
Shaimaa Eliwa Ghazy* and Seham Ahmed Abdel Ghani*
}

\begin{abstract}
Review: Osteosarcoma is considered a primary cancer type of bone neoplasms with locally destructive behavior. Chondrosarcoma is a malignant neoplasm of bone in which there is a production of cartilage matrix by the neoplastic cells. Hedgehog pathway activation is important in osteoblast and chondroblast differentiation. Abnormal activation of the Hh pathway results in malignant neoplasms formation such as stomach cancer, breast, intestinal cancer and prostate cancer. Many researches assume the participation of Hh activation in cancer-related neovascularization. VEGFR2 is a type $\mathrm{V}$ receptor tyrosine kinase which is seen in vascular endothelial cells. The receptor is activated when attached to its (VEGF) ligand, which starts a phosphorylation steps that finally stimulates activation of endothelial cell growth and migration.
\end{abstract}

Aim of study: The current study was performed to examine the immunohistochemical presence of SHH in both osteosarcoma and chondrosarcoma and correlate its presence with angiogenesis, which has a main part in the spread and invasion of cancer.

Material and Methods: Immunohistochemical expression of SHH and VEGFR2 was evaluated in 10 samples of jaw osteosarcoma cases and 10 samples of jaw chondrosarcoma.

Results: Osteosarcoma expression for both SHH and VEGFR-2, was significantly higher compared to chondrosarcoma with non statistically significant positive correlation between $\mathrm{SHH}$ and VEGFR-2 (P-value $>0.001$ ) in both lesions .

Conclusion: The over expression of SHH in both osteosarcoma and chondrosarcoma indicates its important role in carcinogenesis of these tumors. The positive correlation between SHH and VEGFR2 donates the crucial role of SHH in activation of angiogenesis.

KEYWORDS: osteosarcoma, chondrosarcoma,SHH, VEGFR2, angiogenesis

\footnotetext{
* Lecturer of Oral Pathology, Faculty of Dentistry, Ain Shams University.
} 


\section{INTRODUCTION}

As stated by the Global Cancer Statistics 2018, there is a new 18.1 million cancer condition all over the world. By the year 2025 there will be more increase in the cancer burden especially in the developing countries, with more than 20 million cancer conditions is predictable to occur annually ${ }^{[1,2]}$.

Osteosarcoma is considered a primary cancer type of bone neoplasms with locally destructive behavior and elevated metastatic power. Remote metastases of osteosarcoma, like pulmonary metastases, are difficult to control and have a bad prognosis ${ }^{[3,4]}$. Chondrosarcoma is a malignant neoplasm of bone in which there is a production of cartilage matrix by the neoplastic cells, it shows different histopathological and clinical behavior. Chondrosarcoma is considered the second most type of primary bone neoplasms. Chondrosarcomas are classified into low or intermediate types which have low invasive power and high-grade types which have a bad prognosis. Chondrosarcomas are highly resistant to common chemo and radiation treatment. This resistance might be attributed to low mitotic rates and poor drug penetration into the neoplasm microenvironment due to the poor vascularization and the chondroid dense matrix ${ }^{[5,6]}$. The Hedgehog (Hh) family comprises Sonic Hedgehog (SHH), Indian hedgehog (IHH) and Desert Hedgehog (DHH), had been discovered about forty years ago in Drosophila as crucial controller of cells throughout embryogenesis ${ }^{[7,8]}$.

Hedgehog Hh pathway activation is important in osteoblast and chondroblast differentiation from their ancestor during bone formation. Yet, the function of $\mathrm{Hh}$ activation in adult osteoblasts is not explained till now. Many researches demonstrated that $\mathrm{Hh}$ also stimulates osteoclast formation through osteoblast production of parathyroid hormone-related protein (PTHrP), which activates receptor activator of nuclear factor kappa-B ligand
(RANKL) expression through protein kinase A (PKA) and its target transcription factor cAMP response element-binding protein (CREB). So $\mathrm{Hh}$ prompting in mature osteoblasts monitoring both bone formation and resorption ${ }^{[7]}$.

$\mathrm{Hh}$ activation is transported through the membranous proteins, Smoothened (Smo) and Patched1 (Ptch1). In the lack of Hh ligands, Smo signaling efficacy is blocked by Ptch $1{ }^{[9]}$ then, Ptch1 increases and encourages the stimulation of several kinases CK1, PKA, GSK3, which phosphorylate Gli protein (glioma-associated oncogene). This results in full degradation of Gli 1 protein through the proteasome and partial degradation of Gli2 and Gli3. Partially degraded Gli is trans located to the nucleus and acts as a transcriptional repressor for $\mathrm{Hh}$ target genes. This inhibition is eliminated when $\mathrm{Hh}$ ligands bind Ptch1, starting the traditional Hh course in which the $\mathrm{Hh}$ stimulates the phosphorylation of multiple Ser/Thr residues at the carboxy ends of Smo, leading to the emission of Smo inhibition by Ptch, Smo is stimulated on the cell membrane, that eventually promotes the cytoplasmic protein complex to stimulate the $\mathrm{Hh}$ path. The signal is transferred to Gli factor, that will be transmitted intranuclear to controls gene expression implicated in crucial cellular procedure. Target genes of the $\mathrm{Hh}$ activation are linked to cellular growth, cell cycle, stem cell production and cell invasion ${ }^{[10-13]}$.

Because it is a main route controlling cell growth, differentiation and endurance in embryonic life, $\mathrm{Hh}$ pathway is strictly under control, it is negatively controlled through the Ptch1, which works as a crucial "keeper" of Hh signaling efficacy during embryogenesis and adult life. Ptch1 is a famous tumor suppressor. Decreased Ptch1 activity results in the Gorlin syndrome which is associated with many types of neoplasms ${ }^{[14]}$. Abnormal activation of the Hh pathway results in malignant neoplasms formation such as medulloblastoma, bone neoplasms, stomach cancer, lung, breast, intestinal and prostate cancer ${ }^{[15-18]}$. 
Many researches assume that the participation of $\mathrm{Hh}$ activation in cancer-related neovascularization, SHH could allow development of new vessels in a paracrine mode, activating the output of secreted factors. Gli oncogene is considered a primary transcription agent of the $\mathrm{Hh}$ course, results in the increase of the angiogenic factors, cysteinerich angiogenic inducer 61 (CYR61), vascular endothelial growth factor receptor 1(VEGFR1) and $(\text { VEGFR2 })^{[19-22]}$.

VEGFR2 is a type $\mathrm{V}$ receptor tyrosine kinase which is seen in vascular endothelial cells and controlled by the KDR (kinase insert domain receptor) gene. The receptor is activated when attached to its (VEGF) ligand, which starts a phosphorylation steps that finally stimulates intranuclear regulatory genes leading to activation of endothelial cell growth and migration. VEGFR2 is furthermore known as KDR and Flk-1 (fetal liver kinase 1) ${ }^{[23]}$.

Neoplasms growth rely on the proliferation of new blood vessels, which occurs in response to the production of many growth factors by the primary neoplasm and by metastatic spots. VEGF is a homodimeric protein which has a main role in the angiogenesis process, neovascularization, cancer growth and metastases occurrence ${ }^{[24]}$.

Osteosarcoma patients are managed either through surgical removal or chemo agents, but a lot of patients suffer from metastasis to other remote organs following the treatment especially pulmonary metastases which are difficult to treat. Also due to lack of effective treatment for advanced chondrosarcoma cases, the clinical management of chondrosarcomas is exceptionally challenging ${ }^{[16,25,26]}$.

Because of its role in many malignant neoplasms, Hh signaling pathway is considered an important route in cancer exploration and a new hopeful curative approach for inhibiting tumor progression. However, the molecular action of the Hh/Gli pathway in the pathogenesis and progression of osteosarcoma and chondrosarcoma is not fully explored. To our knowledge, few studies were applied to clarify the correlation between SHH and angiogenesis in both osteosarcoma and chodrosarcoma, so the current study was performed to examine the immunohistochemical presence of SHH in both osteosarcoma and chondrosarcoma and correlate its presence with angiogenesis, which has a main part in the spread and invasion of cancer, suggesting that Hh pathway inhibitor could be a novel anti-angiogenic targeted gene therapy in these bone tumors.

\section{MATERIALS AND METHODS}

In the current study, 10 samples of jaw osteosarcomas and 10 samples of jaw chondrosarcomas were selected. All collected from the archive of Oral Pathology Department, National Cancer Institute, Cairo University.Briefly, immunohistochemical staining was performed as follows: wax blocks were cut at four micrometer thickness. Sections were deparaffinized with xylene and rehydrated in graded alcohol. Sections were placed in citrate buffer before the immunostaining steps. The universal kit (Lab Vision, USA) was utilized. Peroxidaseantiperoxidase process using the biotin-streptavidin system was accomplished, 3\% hydrogen peroxide was added to the sections to stop endogenous peroxidase action. The primary antibodies VEGFR2 (Lab Vision, Fermont CA, USA), SHH (Abcam,UK) were added and then incubated overnight at room temperature. After washing with phosphate buffer saline (PBS), the link antibody was added, followed by streptavidin labeling antibody. After rinsing with PBS, diaminobenzidine chromogen was applied to the sections followed by the counterstain. Sections were dehydrated in graded alcohol, applied in xylene and mounted. All the steps for immunohistochemical quantitative evaluation were carried out on photomicrographs captured at a magnification of X40 using image analysis software (Image J, 1.41a, NIH, USA). 
Numerical data were explored for normality by checking the distribution of data and using tests of normality (Kolmogorov-Smirnov and Shapiro-Wilk tests) as well as normality plots (QQ and density plots). For both SHH and VEGFR-2, data were not normally distributed. Thus, Wilcoxon rank sum test was applied. Spearman correlation was used to calculate correlation between samples. Descriptive statistics were described as median and interquartile range. $P$ value less 0.05 was considered statistically significant. R Foundation for Statistical Computing, Vienna, Austria version 3.6.0 was used for the statistical analysis. $\mathrm{R}$ packages used in the analysis were "broom" version 0.5.2 for producing tidy models and "ggpubr" version 0.2.1 for graphs.

\section{RESULTS}

\section{Immunohistochemical Results}

\section{Sonic Hedgehog}

All ten cases of osteosarcoma demonstrated positive SHH immunoreactivity. Most of the neoplastic osteoblast cells showed mainly cytoplasmic expression with some cells with both nuclear and cytoplasmic expression. Also few cells showed membranous reaction. Some cells showed immunonegative reaction. Most of the neoplastic osteoblasts showed granular reaction. The immunopositive cells were scattered throughout the lesion or arranged in form of lobules. There was also osteoid tissue formation which demonstrated immunonegativity (Fig. 1). All ten

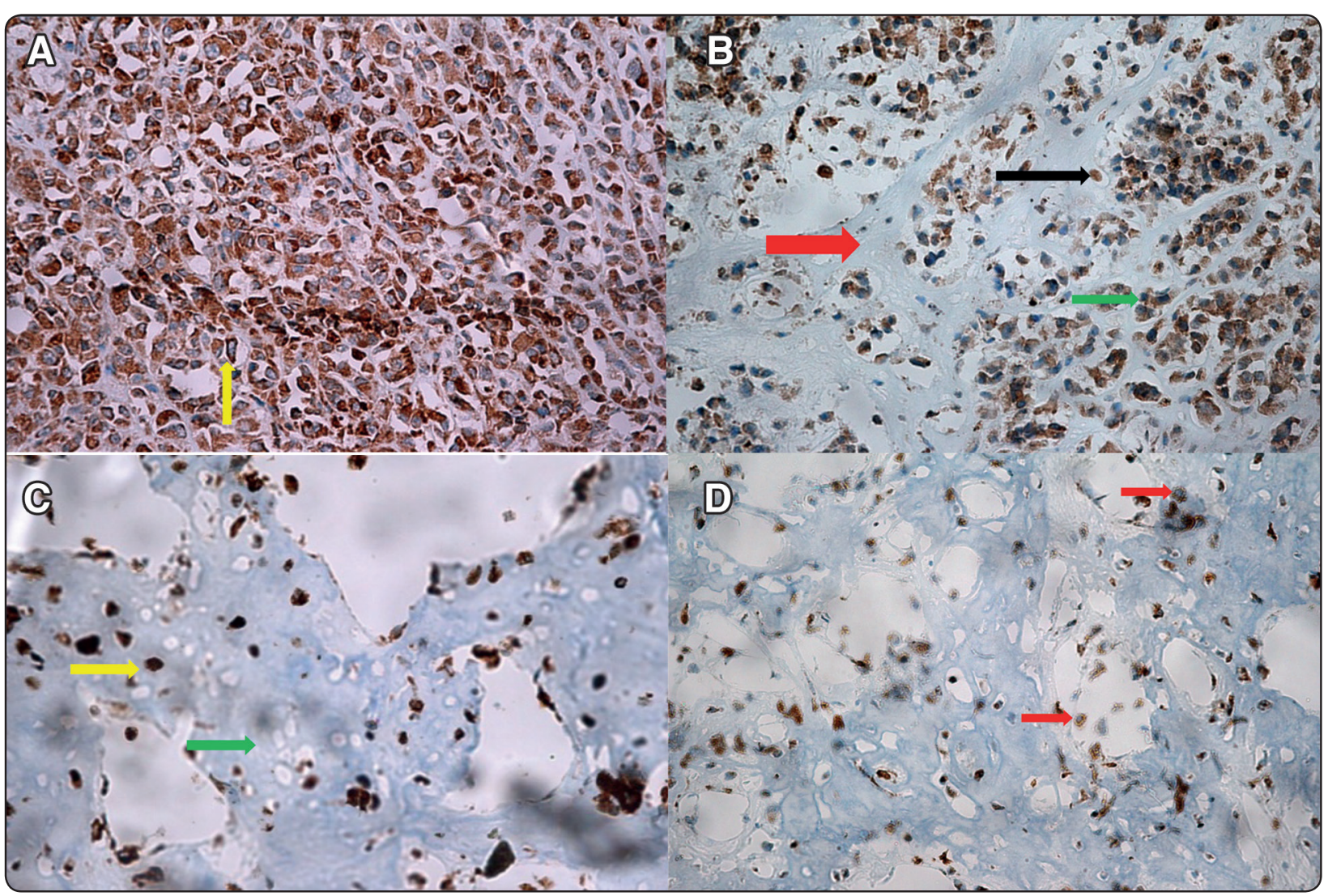

Fig. (1) : Photomicrographs of immunohistochemical results of SHH , in osteosarcoma and chondrosarcoma lesions . A: showing the memberanous expression of SHH in osteosarcoma ( yellow arrow), B: Showing the cytoplasmic reaction of SHH in osteosarcoma (green arrow), cytoplasmic and nuclear reaction( black arrow) and the negative reaction of osseous matrix ( red arrow) C: Showing the nuclear and cytoplasmic reaction in chondrosarcoma ( yellow arrow) and negative cartilage matrix ( green arrow), D: showing the cytoplasmic reaction in chondrosarcoma lesion( red arrow) (Orig. Mag. X40). 
cases of chondrosarcoma showed positive $\mathrm{SHH}$ immune reaction but lesser expression compared to the osteosarcoma lesions. Immune reaction was mainly cytoplasmic and nuclear also some cells were negative. Most of the neoplastic cells showed homogenous reaction. The chondroid matrix showed immunonegative reaction (Fig. 1)

\section{Vascular Endothelial growth factor Receptor-2}

All examined cases of osteosarcoma showed positive VEGFR2 immunopositivity which was seen in neoplastic osteoblasts. The neoplastic cells showed immunopositivity which was either cytoplasmic or both cytoplasmic and nuclear, some of the neoplastic osteoblasts were immunonegative
(Fig. 2). All examined cases of chondrosarcoma revealed VEGFR2 immunoreactivity with variable staining intensity. Immunopositivity was seen in almost all neoplastic chondrocytes. Few cells showed immunonegative reaction. The neoplastic cells revealed cytoplasmic or both cytoplasmic and nuclear reaction. Most of the neoplastic chondrocytes showed a granular or reticular immunostaining, others showed a homogenous reaction (Fig.2).

\section{Statistical results}

The Wilcoxon rank sum test revealed that osteosarcoma expression for bothSHH and VEGFR2, was significantly different from chondrosarcoma $(\mathrm{P}$-value $=0.001)$, were osteosarcoma showed

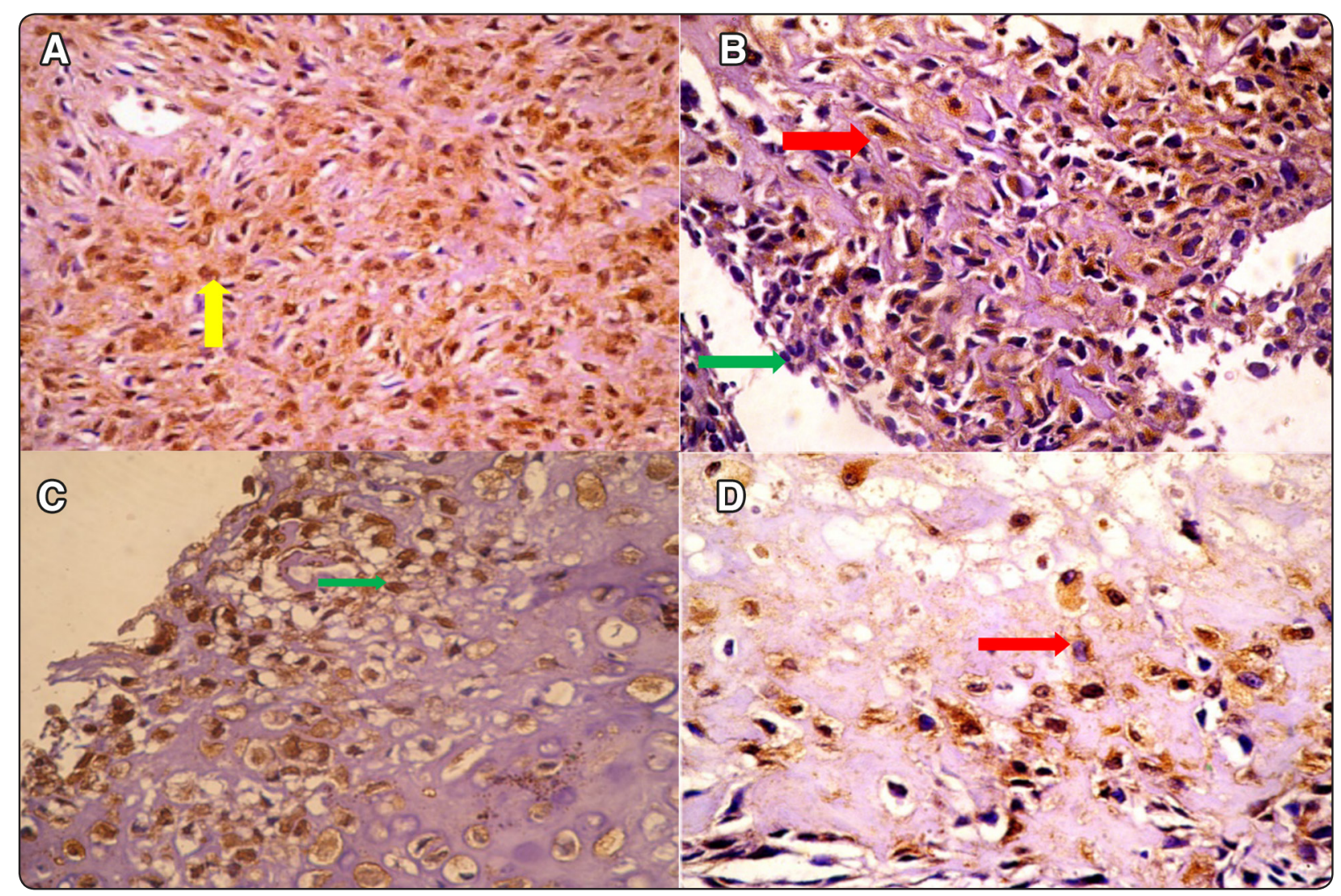

Fig. (2) : Photomicrographs of immunohistochemical results of VEGFR2, in osteosarcoma and chondrosarcoma lesions . A: showing the cytoplasmic and nuclear expression of VEGFR2 in osteosarcoma ( yellow arrow), B: Showing the cytoplasmic reaction in osteosarcoma (red arrow) and some negative cells (green arrow) C: Showing the nuclear and cytoplasmic reaction in chondrosarcoma ( green arrow), D: showing the cytoplasmic reaction in chondrosarcoma lesion( red arrow) (Orig. Mag. X40). 
the highest median value for SHH and VEGFR2 (20.7 and 22.3) respectively while chondrosarcoma showed lower median value for both $\mathrm{SHH}$ and VEGFR2 (7.5 and 9) respectively (table 1, Fig. 3)

\section{Correlation between SHH and VEGFR2}

A statistically non significant positive relation between SHH and VEGFR2 (P-value $>0.001$ ) in both lesion ns osteosarcoma and chondrosarcoma (table 2, Fig. 4).

TABLE (1) : Wilcoxon rank sum test for SHH and VEGFR2 in both osteosarcoma and chondrosarcoma

\begin{tabular}{|l|c|c|c|c|c|}
\hline \multicolumn{1}{|c|}{ Group } & median & IQR & p.value & method & alternative \\
\cline { 1 - 3 } Chondrosarcoma (SHH) & 7.580 & 1.408 & \multirow{2}{*}{0.000} & Wilcoxon rank sum test & two.sided \\
\cline { 1 - 3 } Osteosarcoma (SHH) & 20.758 & 3.677 & \multirow{2}{*}{0.000} & \multirow{2}{*}{ Wilcoxon rank sum test } & two.sided \\
\cline { 1 - 4 } Chondrosarcoma (VEGFR2) & 9.023 & 1.348 & 5.474 & & \\
\hline
\end{tabular}

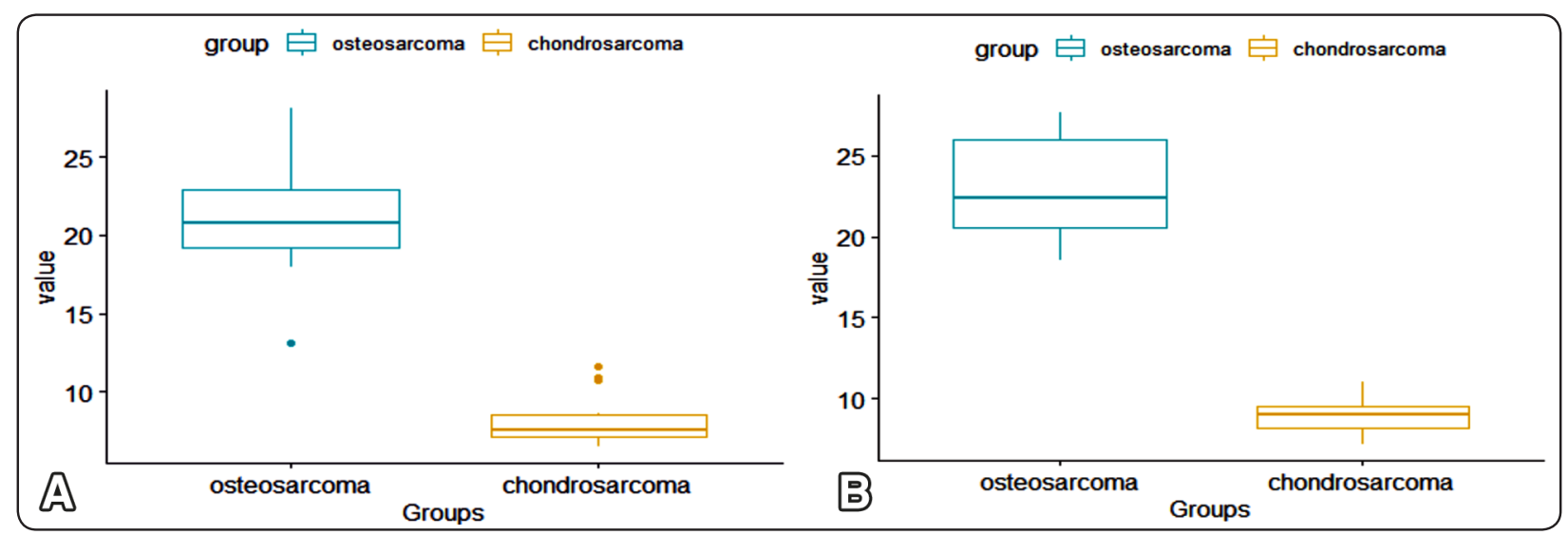

Fig. (3): Box blot showing the mean value of SHH (A) and VEGFR2 (B) in both osteosarcoma and chondrosarcoma

TABLE (2): spearman correlation for SHH and VGFR2 in both osteosarcoma and chondrosarcoma

\begin{tabular}{|ll|l|l|l|}
\hline \multicolumn{2}{|c|}{ Estimate } & \multicolumn{1}{c|}{ p.value } & \multicolumn{1}{c|}{ Method } & \multicolumn{1}{c|}{ Aternative } \\
\hline Osteosarcoma & 0.236 & 0.397 & Spearman's rank correlation rho & two.sided \\
\hline Chondrosarcoma & 0.404 & 0.135 & Spearman's rank correlation rho & two.sided \\
\hline
\end{tabular}




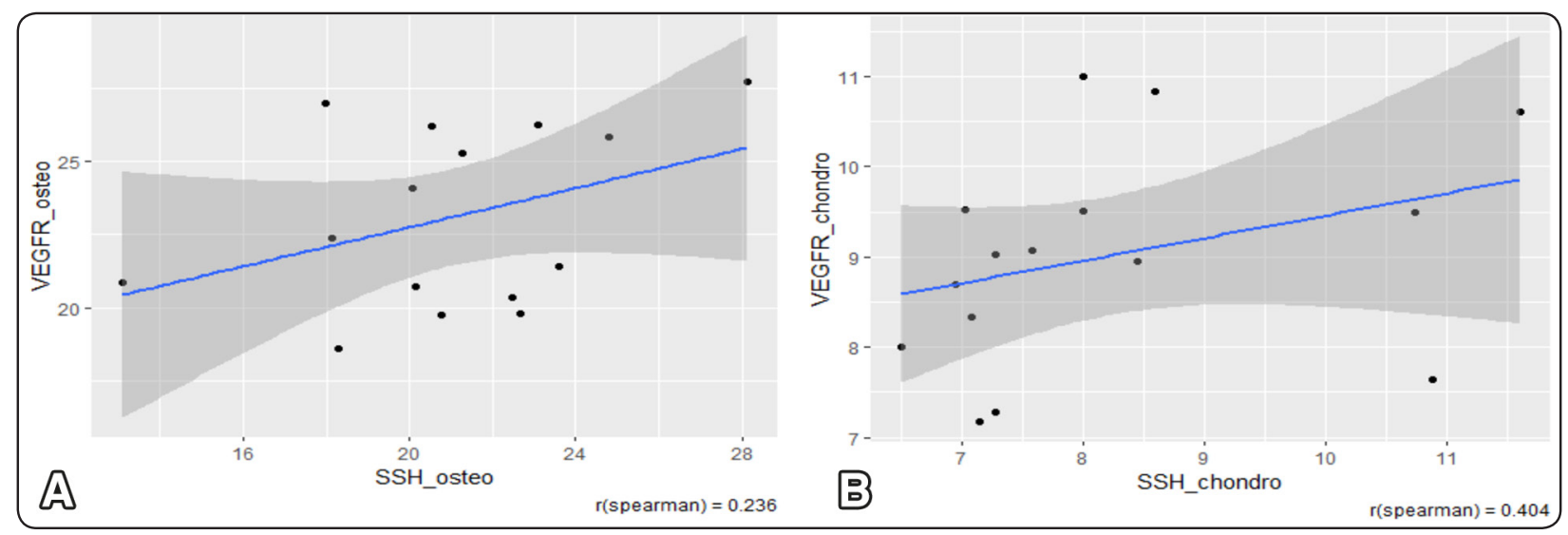

Fig. (4): Scatter diagram representing the correlation between SHH and VEGFR2 in osteosarcoma (A) and chondrosarcoma (B)

\section{DISCUSSION}

Osteosarcoma is considered the most common bone neoplasm with locally aggressive effect and high risk of metastasis followed by chondrosarcoma which has distinct clinical behavior ${ }^{[4,5]}$. The continued need of novel targeted therapeutic treatment became a vital matter to improve the survival rate of patients. In the current study, we examined the SHH expression in both osteosarcoma and chondrosarcoma and correlated its expression with angiogenesis, which has a considerable part in the invasion of cancer. SHH is quiescent in adult cells. When this pathway is stimulated aberrantly in adult cells , it leads to cancer development ${ }^{[27]}$. In this study, SHH was highly expressed in both lesions. This finding was in accordance with previous studies which suggested that SHH might have a noticeable role in occurrence of osteosarcoma and chondrosarcoma ${ }^{[18,29,30]}$.

The mechanisms by which SHH participates in the neoplastic transformation might be through different methods such as stimulation of genes responsible for cell proliferation and survival, increasing the ability of cancer cells to evade from the immune system ${ }^{[31,32]}$, the activation of neovascularization by the production of proangiogenic elements like VEGF and fibroblast growth factors (FGF) ${ }^{[33]}$. Finally, SHH over expression might permit the chemoresistance and radioresistance in different malignant tumors ${ }^{[34]}$.

Our results showed a statistically significant higher expression of $\mathrm{SHH}$ protein in oral osteosarcoma than chondrosarcoma. This might be explained by that higher expression of $\mathrm{SHH}$ relates to the aggressiveness of the tumors indicating the more aggressive path of osteosarcoma cases in the present study, this is in agreement with Srinath et al., $2016^{[27]}$, who found a significant increase in SHH expression in poorly differentiated squamous cell carcinoma than moderately differentiated squamous cell carcinoma. Also, Eefting et al., 2009 ${ }^{[28]}$, mentioned that chondrosarcomas are commonly slowly growing chondrogenic neoplasms of intermediate malignancy and rarely metastasizing. This also might explain the higher SHH expression in our osteosarcoma cases.

The expression of SHH in this study was mainly cytoplasmic, but some neoplastic cells showed other patterns of expression such as nuclear and membranous expression and this was accordance with other studies [27, 35,36,37]. The membranous expression of $\mathrm{SHH}$ could be explained by the fact that the whole $\mathrm{SHH}$ molecule is divided in the cytoplasm into $\mathrm{N}$ - and $\mathrm{C}$ - parts, while the $\mathrm{C}$-terminal end is freely secreted, the $\mathrm{N}$-terminal part is adjusted by lipid hydrophobic alterations and kept in the cell membrane $e^{[38]}$. 
The stroma, osteoid and chondroid tissues in this study demonstrated SHH immunonegativity, this was in contrary to Li-Ma et al., who showed SHH immunopositivity in gastric carcinoma stromal tissues, this was explained by as the cancer cells proceed, they release high amount of SHH in the surrounding stromal tissue resulting in cancer progression $^{[39]}$.

VEGF family has a major role in the angiogenesis procedure, malignant proliferation and propagation $^{[24]}$. In the present study, an increase in VEGFR2 expression was observed in both lesions, this was in accordance with previous studies which reported that VEGFR2 expression was obviously high in osteosarcoma tissues and was associated with poor prognosis. Also Chondrosarcomas as they enlarge, are capable of producing an angiogenic elements and metastasizing to remote regions ${ }^{[40,41]}$.

Higher expression of VEGFR2, may be stimulated fundamentally by elevated levels of HIF $-1 \alpha$ which is a transcription factor generated under the hypoxic conditions and decreased oxygen found in malignant environment. Hypoxia is also recognized to increase CXCR4 expression which in turn activates the tumor advancement through stimulation of angiogenesis and migration ${ }^{[40,42]}$.

Higher expression of VEGFR2 in osteosarcoma cases with statistical significant increase in comparison with chondrosarcoma cases was observed in the present sttudy. This result was in accordance with that have mentioned by Kim et al that VEGFR2 expression level is related with the invasiveness of squamous cell carcinoma ${ }^{[43]}$. This again support the aggressive behavior of osteosarcoma cases in the present study. The expression of VEGFR2, in this study was mainly cytoplasmic or both cytoplasmic and nuclear, this was in accordance with several studies ${ }^{[41,44,45]}$.

The nuclear expression of VEGFR2 suggesting that molecular mechanisms that participate in tumor angiogenesis might need a particular activity of this protein in the nucleus, indicating that nuclear VEGFR2 might magnify the angiogenic response. However, the exact activity of VEGFR2 in the nucleus is unknown ${ }^{[46]}$.

The present study also showed direct positive correlation between the expression of SHH and VEGFR2 which is non-statistically significant. This result is in accordance with what was reported that hyperactivation of $\mathrm{Hh}$ signaling pathway and the ligand $\mathrm{SHH}$ in cancer cells themselves lead to increase proangiogenic factor expression in a paracrine manner, including VEGFR2, matrix metallopeptidase 2, matrix metallopeptidase and heparanase $^{[19,20,47]}$.

\section{CONCLUSION}

The over expression of SHH in both osteosarcoma and chondrosarcoma indicates its important role in carcinogenesis of these tumors. On the other hand the positive correlation between SHH and VEGFR2 donates the crucial role of $\mathrm{SHH}$ in cancer cells metastasis and progression through the activation of angiogenesis which makes it a promising prognostic factor and new anti-angiogenic targeted gene therapy in these aggressive bone tumors.

\section{REFERENCES}

1. Bray F., Ferlay J., Soerjomataram I., Siegel R., Torre L. and Jemal A.: Global cancer statistics 2018: GLOBOCAN estimates of incidence and mortality worldwide for 36 cancers in 185 countries. CA Cancer J Clin. 2018;68::394-424.

2. Ferlay J., Soerjomataram I., Dikshit R., Eser S., Mathers C., Rebelo M., Parkin D., Forman D. Bray F.: Cancer incidence and mortality worldwide: sources, methods and major patterns in GLOBOCAN 2012. Int J Cancer. 2015;136:E359-E386.

3. Wang D., Song Z. and Wang Z.: Common mechanism of pathogenesis in various types of metastatic osteosarcoma. Oncol. Lett.2017; 14:6307-6313 .

4. Hu F., Wang W., Zhou C. and Shang F.: High expression of periostin is dramatically associated with metastatic potential and poor prognosis of patients with osteosarcoma. World J. Surg. Oncol.2014; 12: 1-8. 
5. Bovée J., Cleton-Jansen A., Taminiau A. and Hogendoorn P.: Emerging pathways in the development of chondrosarcoma of bone and implications for targeted treatment. Lancet Oncol. 2005;6:599 - 607 .

6. Van Oosterwijk J., Herpers B., Meijer D. Briaire-de Bruijn I., Cleton-Jansen A., Gelderblom H., van de Water B. and \& Bove' e J.: Restoration of chemosensitivity for doxorubicin and cisplatin in chondrosarcoma in vitro: BCL-2 family members cause chemoresistance. Ann. Oncol. 2012;23: 1617-1626 .

7. Mak K., Bi Y., Wan C., Chuang P., Clemens T., Young M. and Yang Y.: Hedgehog Signaling in Mature Osteoblasts Regulates Bone Formation and Resorptionby Controlling PTHrP and RANKL Expression. Developmental Cell 2008;14: 674-688.

8. Nüsslein-Volhard C., Wieschaus E.: Mutations affecting segment number and polarity in Drosophila. Nature 287;1980: 795-801 .

9. Huangfu, D., and Anderson, K.: Signaling from Smo to Ci/ Gli: conservation and divergence of Hedgehog pathways from Drosophila to vertebrates. Development 2006; 133:3-14.

10. Shi Q., Han Y. and Jiang J.: Suppressor of fused impedes $\mathrm{Ci} /$ Gli nuclear import by opposing Trn/Kapbeta2 in Hedgehog signaling. J. Cell Sci. 2014; 127: 1092-1103

11. Laner-Plamberger S., Wolff F., Kaser-Eichberger A., Swierczynski S., Hauser-Kronberger C. Frischauf A.and Eichberger T.: Hedgehog/GLI signaling activates suppressor of cytokine signaling 1 (SOCS1) in epidermal and neural tumor cells. PLoS One. 2013;8:e75317.

12. Ruiz I., and Altaba A.: Hedgehog signaling and the Gli code in stem cells, cancer, and metastases. Sci. Signal.2011; 4, pt9 .

13. Skoda A., Simovic D., Karin V., Kardum V., Vranic S. and Serman L.:The role of the Hedgehog signaling p.athway in cancer: A comprehensive review. Bosn J Basic Med Sci. $2018 ; 20: 18: 8-20$

14. Rubin, L. and de Sauvage, F.:Targeting the Hedgehog pathway in cancer. Nat. Rev. Drug Discov.2006; 5, 1026-1033.

15. Wu F., Zhang Y., Sun B., McMahon A. and Wang Y. Hedgehog Signaling.: From Basic Biology to Cancer Therapy. Cell Chem Biol. 2017;16:24:252-280.

16. Polychronidou G., Karavasilis V., Pollack S., Huang P., Lee A. and Jones R.: Novel therapeutic approaches in chondrosarcoma. Future Oncol. 2017;13:637-648.

17. Mukherjee S., Frolova N., Sadlonova A., Novak Z., Steg A., Page G., Welch D., Lobo-Ruppert S., Ruppert J., Johnson M.,
Frost A.: Hedgehog signaling and response to cyclopamine differ in epithelial and stromal cells in benign breast and breast cancer. Cancer Biol Ther. 2006 Jun; 5:674-83.

18. Yao Z., Han L., Chen Y., He F., Sun B., Kamar S. and Yang $\mathrm{Z}$.: Hedgehog signalling in the tumourigenesis and metastasis of osteosarcoma, and its potential value in the clinical therapy of osteosarcoma. Cell death \& disease. 2018; 9:2-12.

19. Yamazaki M., Nakamura K. Mizukami Y., Ii M., Sasajima J., Sugiyama Y., Nishikawa T., Nakano Y., Yanagawa N., Sato K., Maemoto A., Tanno S., Okumura T., Karasaki H., Kono T., Fujiya M., Ashida T., Chung D. and Kohgo Y.: Sonic Hedgehog derived from human pancreatic cancer cells augments angiogenic function of endothelial progenitor cells. Cancer Sci 2008; 99: 1131-1138.

20. Harris L., Pannell L., Singh S., Samant R.and Shevde L.: Increased vascularity and spontaneous metastasis of breast cancer by Hedgehog signaling mediated upregulation of cyr61. Oncogene 2012; 31: 3370-3380.

21. Goel H., Pursell B., Chang C., Shaw L., Mao J., Simin K., Kumar P., Vander Kooi C., Shultz L., Greiner D., Norum J., Toftgard R., Kuperwasser C., Mercurio A.: GLI1 regulates a novel neuropilin-2/a6b1 integrin based autocrine pathway that contributes to breast cancer initiation. EMBO Mol Med 2013; 5: 488-508

22. Cao X., Geradts J., Dewhirst M. and Lo H.: Upregulation of VEGF-A and CD24 gene expression by the tGLI1 transcription factor contributes to the aggressive behavior of breast cancer cells. Oncogene 2012; 31: 104-115

23. Shibuya M.: Vascular endothelial growth factor (VEGF)-receptor 2: its biological functions, major signaling pathway, and specific ligand VEGF-E. Endothelium. 2006; 13:63-69.

24. DuBois S. and Demetri G.: Markers of angiogenesis and clinical features in patients with sarcoma. Cancer. 2007;109:813-9.

25. Pratap S., Pokala R., Meyer H., Gehrs C. and Palacios F.: Diffuse marrow involvement in metastatic osteosarcoma: an unusual presentation. J. Oncol. Pract.2017; 13: 401-403

26. Zandueta C., Ormazábal C., Perurena N. Martínez-Canarias S., Zalacaín M., Julián M., Grigoriadis A., Valencia K., Campos-Laborie F., Rivas Jde L., Vicent S., Patiño-García A. and Lecanda F. : Matrix-Gla protein promotes osteosarcoma lung metastasis and associates with poor prognosis. $\mathrm{J}$ Pathol. 2016; 239:438-49.

27. Srinath S., Iyengar A. and Mysorekar V.: Sonic hedgehog in oral squamous cell carcinoma: An immunohistochemical study. J Oral Maxillofac Pathol. 2016;20:377-383. 
28. Eefting D., Schrage Y., Geirnaerdt M., Le Cessie S., Taminiau A., Bovée J., Hogendoorn P., Consortium E.:Assessment of interobserver variability and histologic parameters to improve reliability in classification and grading of central cartilaginous tumors. Am. J. Surg. Pathol. 2009;33:50-57.

29. Qu W., Wang Y., Wu Q., Hao D.and Li D.: Emodin Impairs Radioresistance of Human Osteosarcoma Cells by Suppressing Sonic Hedgehog Signaling. Med Sci Monit. 2017;23:5767-5773.

30. Campbell v., Nadesan P., S. Ali A., Wang C., Whetstone H., Poon R., Wei Q., Keilty J., Proctor J., Wang L., Apte S., McGovern K., Alman B. and Wunder J.: Hedgehog Pathway Inhibition in Chondrosarcoma Using the Smoothened Inhibitor IPI-926 Directly Inhibits Sarcoma Cell Growth. Mol Cancer Ther. 2014 ; 1259-1269.

31. Wilkinson S., Furic L., Buchanan G., Larsson O., Pedersen J., Frydenberg M., Risbridger G., Taylor R..: Hedgehog signaling is active in human prostate cancer stroma and regulates proliferation and differentiation of adjacent epithelium. Prostate. 2013;73:1810-1823.

32. Hanna A. and Shevde L.: Hedgehog signaling: modulation of cancer properies and tumor mircroenvironment Molecular Canc;2016:15:-14.

33. Carmeliet P. and Jain R.: Angiogenesis in cancer and other diseases. Nature 2000;407:249-57.

34. Shahi M., Farheen S., Mariyath M., Castresana J.: Potential role of Shh-Gli1-BMI1 signaling pathway nexus in glioma chemoresistance. Tumour Biol. 2016;37: 15107-15114.

35. Al Ghamdi D., Gomaa W., Abulaban A., Al-Ahwal M., Buhmeida A., Al-Qahtani M. and Al-Maghrabi J.: The significance of sonic hedgehog immunohistochemical expression in colorectal carcinoma. J. Microsc Ultrastruct. 2015; 3:169-174.

36. Song X., Yan L., Lu C., Zhang C., Zhu F., Yang J., Jing H., Zhang Y., Qiao J.and Guo H.: Activation of hedgehog signaling and its association with cisplatin resistance in ovarian epithelial tumors. Oncol Lett. 2018;15: 5569-5576

37. Leprieur E., Tolani B.,Li H., Leguay F., Hoang N., Acevedo L.,Jin J., Tseng Hh., Yue D.,Kim I., Wislez M.,Wang C., Jablons D. and He B.: Membrane-bound full-length Sonic Hedgehog identifies cancer stem cells in human nonsmall cell lung cancer. Oncotarget 2017;8:103744-103757.
38. Noman A., Uddin M., Rahman M., Nayeem M., Alam S., Khatun Z., Wahiduzzaman M., Sultana A., Rahman M., Ali M., Barua ., Ahmed I., Islam M., Aboussekhra A., Yeger H., Farhat W. and Islam S.: Overexpression of sonic hedgehog in the triple negative breast cancer: clinicopathological characteristics of high burden breast cancer patients from Bangladesh. Sci Rep.2016;6:1-11

39. Li Ma X., Sun . Wang Y., Huang S., Xie J., Zhang H.: Study of Sonic hedgehog signaling pathway related molecules in gastric carcinoma. World J. Gastroenterol. 2006;12:3965-3969.

40. Sun X., Wei L., Chen Q., and Tere, R.: CXCR4/SDF1 mediate hypoxia induced chondrosarcoma cell invasion through ERK signaling and increased MMP1 expression. Mol. Cancer 2010;9: 1-11

41. Liu K., Ren T. Huang Y., Sun K., Bao X., Wang S., Zheng B. and GUO W.: Apatinib promotes autophagy and apoptosis through VEGFR2/STAT3/BCL-2 signaling in osteosarcoma. Cell Death \& Disease. 2017; 8: 1-10

42. Schioppa T., Uranchimeg B., Saccani A., Biswas S., Doni A., Rapisarda A., Bernasconi S., Saccani S., Nebuloni M., Vago L., Mantovani A., Melillo G. and Sica A.: Regulation of the chemokine receptor CXCR4 by hypoxia. J Exp Med 2003, 198:1391-1402.

43. Kim S., Park S., Kim K.: Expression of vascular endothelial growth factor in oral squamous cell carcinoma. J Korean Assoc Oral Maxillofac Surg. 2015;41:11-18.

44. Miettinen M., Rikala M., Rys J., Lasota J., Wang Z.: Vascular endothelial growth factor receptor 2 as a marker for malignant vascular tumors and mesothelioma: an immunohistochemical study of 262 vascular endothelial and 1640 nonvascular tumors. Am J Surg Pathol. 2012;36:629-39.

45. Lian, L., Li, X., Xu M., Li X., Wu M., Zhang Y., Tao M., Li W., Shen X., Zhou C. and Jiang M.: VEGFR2 promotes tumorigenesis and metastasis in a pro-angiogenic-independent way in gastric cancer. BMC Cancer 2019;19:2-15.

46. Domingues I., Rino J. Demmers J., de Lanerolle P. Santos S.: VEGFR2 translocates to the nucleus to regulate its own transcription. PLoS One. 2011;6:e25668.

47. Di Mauro C., Rosa R., D’ Amato V., Ciciola P., Servetto A., Marciano R., Orsini R., Formisano L., De Falco S., Cicatiello V., Di Bonito M., Cantile M., Collina F., Chambery A., Veneziani B., De Placido S. and Bianco R.: Hedgehog signalling pathway orchestrates angiogenesis in triplenegative breast cancers. Br J Cancer. 2017;116:1425-1435 . 